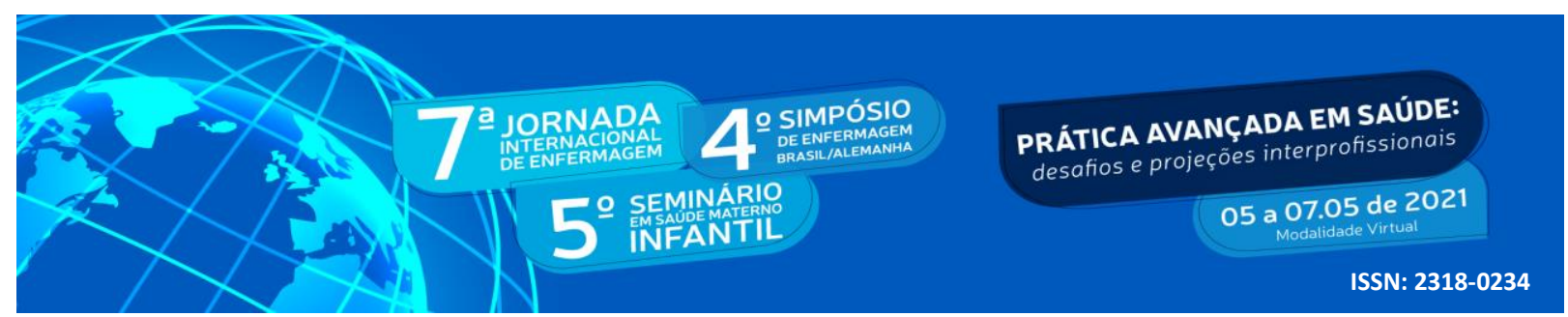

DOI: http://doi.org/10.48195/jie2021-170

\title{
PLANEJAMENTO DA ASSISTÊNCIA DE ENFERMAGEM À GESTANTE EM UMA UTI COVID $^{1}$
}

\section{Fabricio da Cunha Moraes ${ }^{2}$; Bruna de Oliveira Jochims ${ }^{3}$;Caroline Santini Rauber ${ }^{4}$; Evelyne Duarte de Amorim Silva 5 .}

\author{
RESUMO
}

A Organização Mundial de Saúde (OMS) definiu 2020 como o ano da enfermagem, porém a pandemia causada pelo vírus SARSCoV-2 trouxe com ele um grande desafio, o de adaptação e cuidado holístico ao paciente em meio ao caos do sistema de saúde mundial. Nesse contexto, ainda que falte mais evidências quanto à gravidade do COVID-19 para gestantes, a OMS as classificou como grupo de risco. O objetivo deste Relato de Experiência (RE) é demonstrar que a Sistematização da Assistência de Enfermagem (SAE) pode ser o diferencial para pacientes em tratamento hospitalar devido a complicações relacionadas a COVID-19. A vivência aconteceu no período de 14/11/2020 à 23/11/2020, devido a internação de uma gestante relacionado às complicações do COVID-19 numa Unidade de Terapia Intensiva de um hospital de Porto Alegre - RS, com a adaptação do atendimento para desfecho gestacional na UTI pela equipe do Centro Cirúrgico Obstétrico (CCO).

Palavras-chave: Covid-19; Enfermagem; Saúde Materno-Infantil; Obstetrícia.

\begin{abstract}
The World Health Organization (WHO) defined 2020 as the year of nursing, but the pandemic caused by the SARSCoV-2 virus brought a huge challenge of adaptation and holistic patient care among the chaos of the world health system. Even though there is a lack of further evidence about the severity of COVID-19 for pregnant women, the WHO has classified them as a risk group. The objective of this Experience Report (ER) is to demonstrate that the Systematization of Nursing Care (SNC) can be the differential for patients in hospital treatment due to complications related to COVID-19. The experience happened in the period from $11 / 14 / 2020$ to $11 / 23 / 2020$, during the hospitalization of a pregnant woman

\footnotetext{
${ }^{1}$ Relato de experiência.

${ }^{2}$ Enfermeiro Supervisor do Centro Cirúrgico Obstétrico. E-mail: fabricio.enfo@gmail.com

3 Bruna de Oliveira Jochims. Irmandade Santa Casa de Misericórdia de Porto Alegre. E-mail: brujochims@ hotmail.com

4 Caroline Santini Rauber. Irmandade Santa Casa de Misericórdia de Porto Alegre. E-mail:carolinesantini@hotmail.com

5 Evelyne Duarte de Amorim Silva. Irmandade Santa Casa de Misericórdia de Porto Alegre. E-mail: evelyneduarte.silva@gmail.com
} 


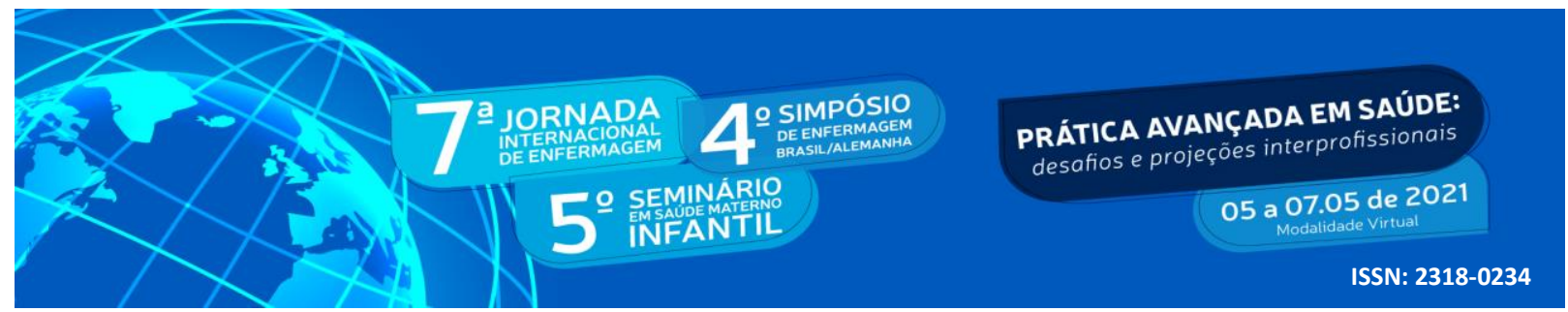

related to complications of COVID-19 in an Intensive Care Unit of a hospital in Porto Alegre - RS, with adjustment of care for gestational outcome in the ICU by the team of the Obstetric Surgical Center (OCC).

Key Words: Covid-19; Maternal and Child Health; Nurse; Obstetrics.

\section{INTRODUÇÃO}

Desde de dezembro de 2019 o mundo vem se adaptando a uma nova realidade devido a uma infecção causada pelo novo Coronavírus, conhecido pela sigla COVID-19 (coronavírus disease 2019), reconhecido como pandemia em março pela Organização Mundial da Saúde em março de 2020 (OMS, 2020).

O Sars-Cov-2, o agente etiológico responsável pela COVID-19, é um vírus respiratório capaz de causar pneumonia e progredir rapidamente para Síndrome Respiratória Aguda Grave (SRAG), com alto potencial de disseminação e contágio (YANG; WANG; POON, 2020). Seu diagnóstico acontece através da presença dos sintomas relacionados à doença como febre, perda de palato, perda de olfato, dor no corpo, exposição conhecida ou, ainda, através da realização do teste para Sars-Cov-2, mesmo na ausência de sintomas (RCOM, 2021).

Segundo estudos, o vírus da SARSCoV-2 pode ser transmitido através do contato próximo de pessoas infectadas pelo vírus. $\mathrm{O}$ vírus pode se espalhar através de gotículas ou aerossóis disseminadas através da boca e nariz da pessoa infectada quando ela tosse, espirra ou até mesmo fala. Segundo a OMS, o contato próximo pode expor o profissional de saúde a uma maior suscetibilidade de se contaminar com o vírus, uma vez que o contato direto e constante aumenta o risco de exposição a essas partículas (WHO, 2021).

Como forma de buscar proteger a população da exposição ao vírus e as possíveis consequências, a OMS definiu alguns grupos de risco, ou seja, aqueles que devem receber um olhar mais atento e, onde as gestantes foram incluídas. Embora ainda não existam evidências científicas que apontem que gestantes possuem um maior risco de contaminação, as alterações na imunológicas e fisiológicas na gestação tornam as gestantes mais propensas a infecções virais, deixando vulnerável também o feto (YANG; WANG; POON, 2020). 


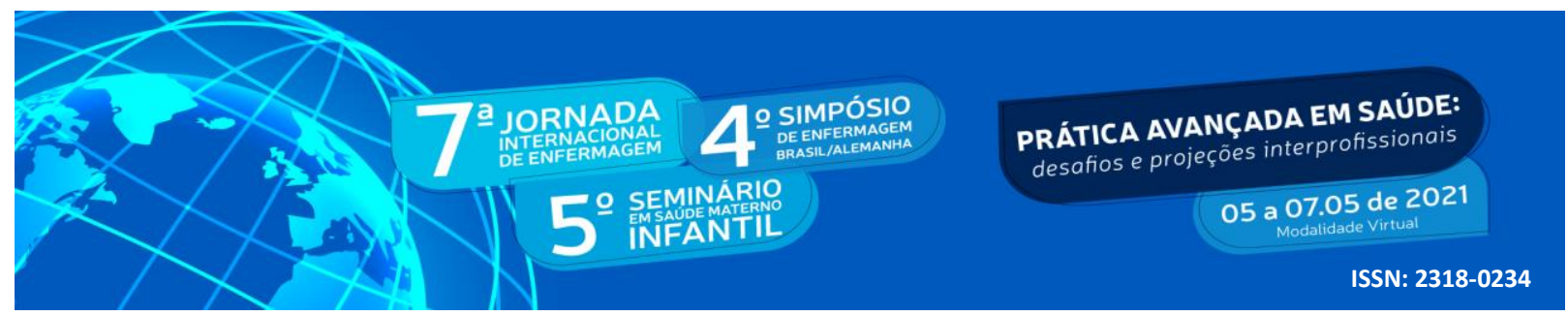

Ainda pouco se sabe sobre as manifestações clínicas da COVID-19 na gestação, estimase que as manifestações sejam semelhantes para adultos não grávidos, porém cada caso deve ser avaliado, levando em consideração a idade gestacional e o bem estar materno e fetal (YANG; WANG; POON, 2020). Chen H et al. (2020) observaram como sintomas prevalentes em gestantes positivas para COVID-19, tosse e febre, além de sintomas menos comuns como dor no corpo, mal estar, dor de garganta e sintomas gastrointestinais.

Embora não seja algo comum, o número de gestantes com COVID-19 no mundo todo vem aumentando a preocupação das autoridades de saúde tanto quanto ao risco para a gestante quanto ao risco de transmissão vertical do SARS-CoV-2, seja intra-útero, intraparto ou no período neonatal (WHO, 2021). Segundo a OMS (2021), alguns estudos já demonstram que cerca de $1.9 \%$ (95/4907) dos recém-nascidos testaram positivo logo nas primeiras 24 horas de vida, contudo com manifestações clínicas leves.

Frente a essa situação desafiadora, a enfermagem tem um importante papel no manejo da assistência do binômio mãe-bebe, a fim de prestar um cuidado integral. A enfermeira, por sua vez, possui um papel essencial no que diz respeito ao planejamento e gerência das demandas à assistência prestada nos casos de gestantes positivas para COVID-19, realizando a Sistematização da Assistência de Enfermagem (SAE), a organização dos fluxos de pessoal e assistenciais, a fim de prever possíveis intercorrências (VENTURI-SILVA JMA et al., 2020).

Além disso, é papel da enfermeira o treinamento e capacitação da equipe assistencial quanto a utilização correta dos Equipamentos de Proteção Individual (EPI) pelos profissionais de assistência, garantindo assim a proteção dos profissionais de saúde, bem como um atendimento mais qualificado às gestantes e puérperas em geral (VENTURI-SILVA JMA et al., 2020).

Tendo em vista os desafios enfrentados durante a pandemia no que diz a assistência à gestante/puérpera acometida pelo COVID-19 e da importância do planejamento da assistência de enfermagem, acredita-se que esse relato de experiência possa servir como instigador para outras equipes de que é possível prestar um cuidado individualizado e integral à gestante/puérperas que encontram-se hospitalizadas para tratamento da infecção pelo COVID19. 


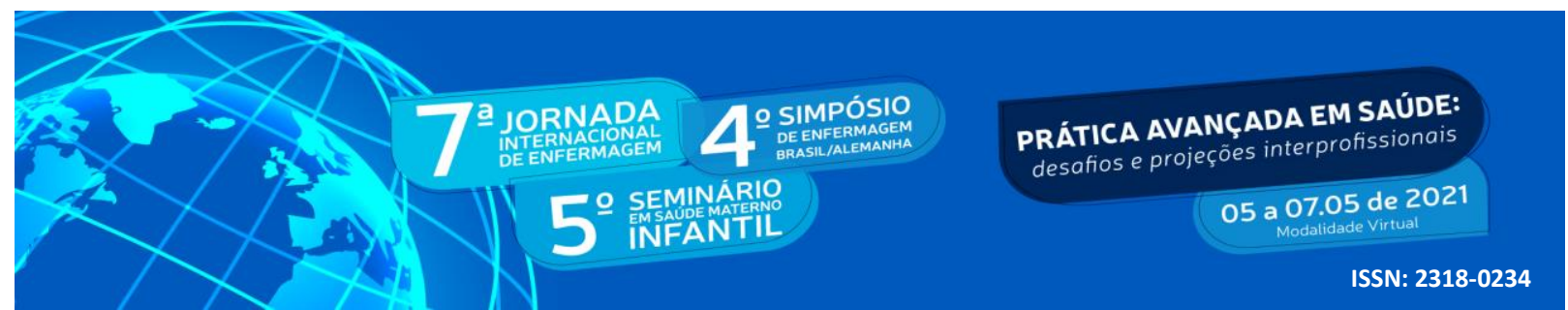

\section{OBJETIVO}

Relatar a experiência da assistência de enfermagem do Centro Obstétrico à uma paciente COVID-19 internada em uma Unidade de Terapia Intensiva (UTI).

\section{METODOLOGIA}

Trata-se de um estudo qualitativo descritivo, na modalidade de Relato de Experiência, que busca descrever uma vivência profissional com o intuito de contribuir para sua área de atuação, resultando em um aperfeiçoamento no cuidado em saúde (DALTRO M.R., FARIA A.A, 2019).

\section{DESENVOLVIMENTO}

Paciente A.O.C, 34 anos, gestante, G3P1C2, IG:27+5, previamente hígida, oriunda de Tramandaí e transferida para um Hospital de Porto Alegre no dia 14/11/20, retaguarda para tratamento de pacientes COVID-19. Segundo informado em nota de transferência iniciou com tosse, febre e dispneia em 11/11/2021 após contato com o esposo suspeita COVID 19, com piora do quadro a partir do dia 13/11/20. Teste rápido para COVID 19 coletado em 14/11/ 2020, resultado positivo. A paciente desenvolveu Pré Eclâmpsia (PE) durante a internação, e evoluiu para cesárea à beira leito na Unidade de Terapia Intensiva (UTI) em 17/11/20, por piora importante do padrão respiratório devido quadro de Síndrome Respiratória Aguda Grave (SRAG).

Embora as gestantes não pareçam estar mais propensas ao contágio pela COVID-19 do que outros adultos, e, quando acometidas, raramente desenvolvem formas graves que necessitem de UTI e ventilação mecânica, sabe-se que o estado gestacional causa alterações fisiológicas na função cardiopulmonar, como aumento de débito cardíaco e diminuição da capacidade pulmonar, tanto por ação hormonal quanto mecânica. Essas alterações tornam a 


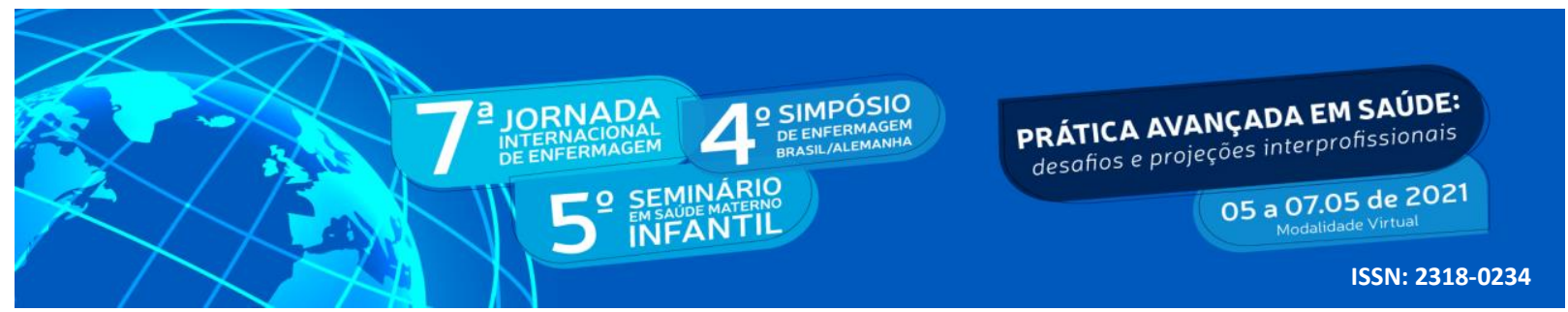

gestante mais suscetível a quadros graves por infecções respiratórias (CHEN H et al. 2020; YANG, H; KOTLAR B et al., 2020; WANG, C; POON, LC 2020).

Por se tratar de um vírus relativamente novo, ainda estão sendo descobertos os impactos da COVID-19 no curso da gestação, no entanto, sabe-se que gestantes acometidas pela patologia estão mais predispostas a desenvolver pré eclâmpsia, trabalho de parto prematuro, ruptura prematura de membranas, cesariana de urgência, prematuridade, restrição de crescimento uterina e óbito tanto materno quanto neonatal (ALLOTEY J et al., 2020)

A Sistematização da Assistência de Enfermagem é uma tarefa privativa de enfermeira e ocorreu de forma ágil e síncrona entre equipe da UTI COVID-19 e Centro Cirúrgico Obstétrico (CO) provando que a assistência bem planejada contribui para uma melhor assistência mesmo quando fora do habitual. Para realizar esse cuidado foi necessário o deslocamento de dois técnicos de enfermagem e uma enfermeira do CO para dar suporte à cesariana e atendimento ao recém-nascido dentro do box onde a paciente estava internada em uma UTI COVID.

Visando a segurança do paciente e prevendo possíveis falhas que poderiam gerar intercorrências, todos os passos do procedimento foram ensaiados com a equipe de obstetras e de neonatologistas, a fim de que todo o material, medicamentos e equipamentos necessários para a realização do procedimento e posterior transporte do RN para UTI Neonatal estivessem disponíveis e fossem deslocados pela equipe de enfermagem à UTI COVID 19 sem sobrecarga.

No que tange ao procedimento em si, o mesmo foi realizado de forma inédita em box de UTI devido a instabilidade materna sob os cuidados da equipe do $\mathrm{CO}$, lançando mão de todos os equipamentos de proteção individual (EPI's) utilizados durante a assistência a pacientes acometidos pela COVID 19. O cuidado foi planejado de forma integral, prevendo-se inclusive a necessidade de ordenha manual de alívio do seio materno realizada pela enfermeira obstetra diariamente.

\section{CONCLUSÃO}

O estudo mostrou que a SAE é processo importante e indispensável frente às situações 


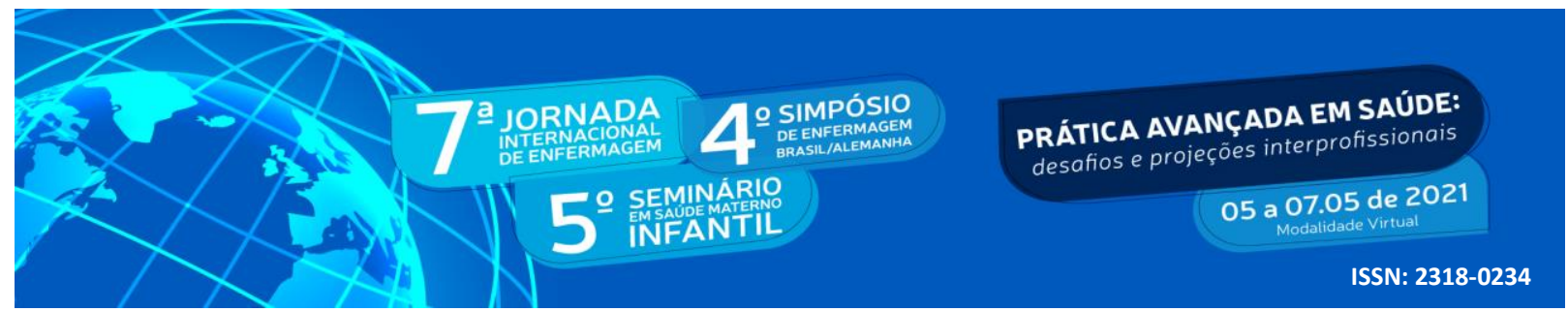

em que seja necessário precisão e agilidade, sendo a enfermeira responsável pelo planejamento das ações, estando a frente do processo. Embora as evidências apontem que gestantes não pareçam ser mais suscetíveis à infecção pela COVID-19, as alterações gestacionais as colocam em grupo de risco, podendo evoluir para quadros que necessitem de maiores cuidados e planejamento de assistência eficaz. Ressalta-se a necessidade de maiores estudos neste campo a fim de aprimorar a assistência a pacientes em UTI que necessitam de intervenção da equipe do Centro Cirúrgico Obstétrico enquanto a pandemia de COVID perdurar, podendo ser adaptado e desenvolvido com outras pacientes que necessitem de internação clínica em UTI durante a gestação ou puerpério.

\section{REFERÊNCIAS}

Allotey, J. et al.. Clinical manifestations, risk factors, and maternal and perinatal outcomes of coronavirus disease 2019 in pregnancy: Living systematic review and meta-analysis. BMJ, 370. https://doi.org/10.1136/bmj.m3320

Chen H. et al., Clinical characteristics and intrauterine vertical transmission potential of COVID-19 infection in nine pregnant women: a retrospective review of medical records. Lancet 2020. https://doi.org/10.1016/ S0140-6736(20)30360-3.

DALTRO, Monica Ramos; FARIA, Anna Amélia de. Relato de experiência: Uma narrativa científica na pós-modernidade. Estudos e Pesquisa em Psicologia, Salvador, v. 1, n. 9, p. 1-1, abr. 2019.

KOTLAR B. et al., The Impact of the COVID-19 Pandemic on Maternal and Perinatal Health: A Scoping Review. Research Square. [s/n]. https://doi.org/10.21203/rs.3.rs-96736/v1

ORGANIZAÇÃO MUNDIAL DA SAÚDE. Respondendo à disseminação do COVID-19 pela comunidade: Orientação provisória. 2020, 1-6.

RCOM. Royal College of Midwives. Coronavirus (COVID-19) Infection in Pregnancy. v 13, n.1, p. 1-98, 2021. Accessed 2021 Feb 20.

VENTURA-SILVA, JMA et al. Planejamento organizacional no contexto de pandemia por COVID-19: implicações para a gestão em enfermagem. Journal Health Npeps, [S.L.], v. 5, n. 1, p. 1-5, 29 jun. 2020. Universidade do Estado do Mato Grosso - UNEMAT. http://dx.doi.org/10.30681/252610104626.

Yang, H.; Wang, C.; Poon, L. C. (2020). Novel coronavirus infection and pregnancy. Ultrasound in Obstetrics \& Gynecology. doi:10.1002/uog.22006 


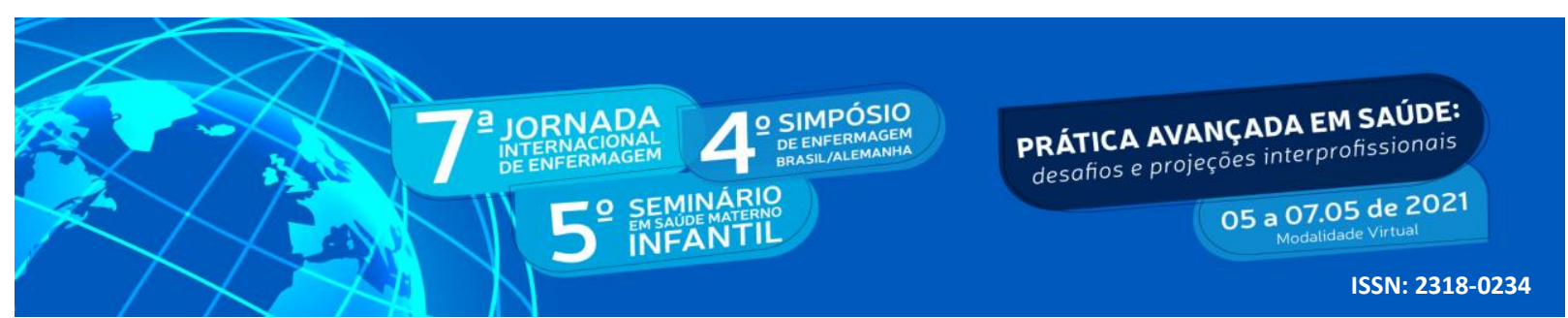

WORLD HEALTH ORGANIZATION et al. COVID19: Occupational health and safety for health workers. Interim guidance, 2 February 2021. World Health Organization, 2021.

WORLD HEALTH ORGANIZATION et al. Definition and categorization of the timing of motherto-child transmission of SARS-CoV-2: scientific brief, 8 February 2021. World Health Organization, 2021. 